\title{
Arbeitnehmerbeteiligung in der Europäischen Aktiengesellschaft (SE) - Eine empirische Analyse der ersten Fälle
}

\begin{abstract}
Seit Herbst 2004 können Unternehmen die neue Rechtsform der Europäischen Aktiengesellschaft (SE) wählen, die gerade aus deutscher Perspektive zunehmend relevant wird. Eine wesentliche Änderung im Vergleich zu den nationalen Rechtsformen besteht darin, dass keine inhaltlich-materiellen Vorgaben zur Beteiligung der Arbeitnehmer gemacht werden. Allerdings muss dieser Punkt - das ist verpflichtend - verhandelt werden, bevor eine SE ins Handelsregister eingetragen werden kann. Das Aushandlungsergebnis ist grundsätzlich offen. Der Beitrag untersucht aus empirischer Perspektive die ersten SEs und vor allem die Verhandlungen und die Ausgestaltung der Arbeitnehmerbeteiligung. Gehen von der SE möglicherweise Risiken für die deutsche Unternehmensmitbestimmung aus? Wird sie zur Vermeidung von Mitbestimmung eingesetzt?
\end{abstract}

\section{Einleitung}

\subsection{KURZER HISTORISCHER ABRISS}

Die Europäische Aktiengesellschaft (Societas Europaea, SE) hat eine lange Geschichte. Bereits Ende der 1950er Jahre gab es erste Vorschläge zur Schaffung einer einheitlichen Unternehmensrechtsform für die damalige Europäische Wirtschaftsgemeinschaft. Nachdem dieses Projekt über Jahrzehnte mit unterschiedlicher Intensität verfolgt wurde und bisweilen ganz von der politischen Agenda verschwunden war, gelang erst mit dem 1997 vorgelegten Bericht der von der Kommission eingesetzten „Davignon"-Gruppe die Auflösung des kritischsten Diskussionspunktes.

Die nationalen Systeme von Arbeitnehmerbeteiligung der damals 18 Mitgliedstaaten des Europäischen Wirtschaftsraumes (EWR) waren auf einen gemeinsamen Nenner zu bringen. Die „Davignon“-Gruppe schlug eine verpflichtende Arbeitnehmerbeteiligung vor, die eine in „uneingeschränkter Freiheit ausgehandelte Lösung" darstellen und auf die ,jeweiligen kulturellen Unterschiede zugeschnitten" sein sollte. Eine solche „flexible“ Lösung sollte Vorrang haben vor „Auffangregelungen“, die nur im Falle eines Scheiterns von Verhandlungen zwischen Arbeitgeber und Arbeitnehmer greifen sollten (Sachverständigengruppe 1997). Ähnlich wie bereits im Falle der Richtlinie „über die Einsetzung eines Europäischen Betriebsrates [...]“ (EBR)
(94/95/EG) wurden lediglich prozedurale Vorgaben, nicht jedoch detaillierte materiell-inhaltliche Regelungen vorgeschlagen (Keller 2002).

Dies war der Schlüssel, um die notwendige Einstimmigkeit zu erreichen. Die „Verordnung des Rates über das Statut der Europäischen Gesellschaft" (EG/2157/2001) (nachfolgend: SE-VO) sowie die „Richtlinie des Rates zur Ergänzung des Statuts der Europäischen Gesellschaft hinsichtlich der Beteiligung der Arbeitnehmer" (2001/ 86/EG) (nachfolgend: SE-RL) traten zum 8. Oktober 2001 in Kraft und mussten bis zum 8. Oktober 2004 in nationales Recht umgesetzt werden. Nachdem sich dieser Prozess in manchen Mitgliedstaaten verzögerte (EWCs Bulletin 2006), können inzwischen Unternehmen in allen EWR-Staaten diese neue Rechtsform nutzen, die eine zusätzliche Option zu den fortbestehenden nationalen Rechtsformen darstellt.

\subsection{PROBLEMSTELLUNG}

Verschiedene Unternehmen haben mittlerweile von der Möglichkeit einer SE-Errichtung Gebrauch gemacht. Im Gegensatz zur umfangreichen Literatur, die sich mit historischen (Sorge 2006), betriebswirtschaftlichen (Theisen/Wenz 2005) und juristischen Fragestellungen (Nagel et al. 2005; Blanke/Köstler 2006) beschäftigt, präsentiert dieser Artikel eine Untersuchung aller bis dato existierenden SEs. Der Fokus liegt auf Fragen der Arbeitnehmerbeteiligung. Eine solche Analyse ist umso wichtiger, als die ersten Fälle ein „Referenzmodell“ (Röthig 2006) für alle weiteren darstellen.
In methodischer Hinsicht basiert dieser Artikel auf einer Analyse der existierenden Literatur, einschließlich des rapide wachsenden ,grauen Marktes“.2 Ferner wurden teilstrukturierte Experteninterviews sowie eine teilnehmende Beobachtung der Ver-

\footnotetext{
1 Die Autoren danken Roland Köstler für wertvolle Hilfe sowie zwei anonymen Gutachtern für ihre weiterführenden Anmerkungen zu der Rohfassung dieses Artikels. Die empirische Forschungsarbeit wurde von der Hans-Böckler-Stiftung gefördert im Rahmen des Promotionskollegs "Arbeitnehmerinteressen und Mitbestimmung in einem Europäischen Sozialmodell“. Unter http://www. sozialmodell.eu finden sich weitere Informationen sowie die Langfassung eines Arbeitspapiers, auf dem dieser Artikel beruht, mit zusätzlichen Tabellen und Abbildungen.

2 Eine wichtige Ressource stellt das "SEEurope-Network" des EGI und der HBS dar. Unter http:// www.worker-participation.eu finden sich viele Informationen und Unterlagen.
}

Berndt Keller, Prof. Dr., lehrt Arbeits- und Sozialpolitik an der Universität Konstanz. Arbeitsschwerpunkte: Arbeitspolitik des öffentlichen Sektors, Folgeprobleme der europäischen Integration, Flexicurity, Atypische Beschäftigung, Zusammenschlüsse von Gewerkschaften.

e-mail: Berndt.Karl.Keller@uni-konstanz.de Frank Werner, Universität Konstanz, Lehrstuhl Prof. B. Keller, Doktorand im Promotionskolleg "Arbeitnehmerinteressen und Mitbestimmung in einem Europäischen Sozialmodell" der Hans-Böckler-Stiftung. e-mail: Frank.Werner@uni-konstanz.de. 
handlungen in einem prominenten Fall durchgeführt.

Der Artikel gliedert sich wie folgt: In Abschnitt 2 geben wir zunächst einen Überblick über die bisher gegründeten SEs und ihre Charakteristika. Danach richten wir den Fokus auf Fragen der Arbeitnehmerbeteiligung. Wir gehen zunächst auf Probleme ein, die die Verhandlungslösung mit sich bringt, wobei Implikationen für die Arbeitnehmerbeteiligung sich bereits aus dem institutionellen Arrangement ergeben (Abschnitt 3). Die Abschnitte 4 und 5 behandeln detailliert die beiden Ebenen von Arbeitnehmerbeteiligung, d.h. die Ebene des sogenannten „SE-Betriebsrats“ bzw. die der Arbeitnehmerbeteiligung in den Leitungsorganen. Ein Ausblick reflektiert Auswirkungen auf die Arbeitsbeziehungen (Abschnitt 6).

\section{Bisherige SE-Gründungen}

\subsection{SUBTYPEN}

Ein grenzüberschreitendes Element ist zur Errichtung einer SE grundsätzlich notwendig. Sie kann auf vier Wegen erfolgen (Art. 2 SE-VO), wobei jede Gründungsform spezifische Implikationen für die Ausgestaltung der Arbeitnehmerbeteiligung hat (vgl. Abschnitt 3 und Köstler 2006):

- durch eine Verschmelzung von Aktiengesellschaften aus zwei Mitgliedstaaten, - durch die Gründung einer Holding-SE von Unternehmen (z. B. AG und $\mathrm{GmbH}$ ) aus zwei Mitgliedstaaten,

- durch Gründung einer Tochter-SE von Gesellschaften oder juristischen Personen öffentlichen oder privaten Rechts aus zwei Mitgliedstaaten (oder durch Gründung einer Tochter-SE durch eine bereits bestehende SE),

- durch Umwandlung einer bestehenden Aktiengesellschaft in eine SE, wenn diese seit mindestens zwei Jahren eine Tochtergesellschaft in einem anderen Mitgliedstaat hat.

Mit Stand Mitte 2007 sind fast 90 SEs in die nationalen Handelsregister eingetragen (SEEurope-Network 2007). ${ }^{3}$ Es handelt sich jedoch nicht ausschließlich um Unternehmen, die sowohl wirtschaftliche Akti- vität als auch Arbeitnehmer aufweisen. Vielmehr sind „Subtypen“ mit ungewöhnlichen Charakteristika zu identifizieren (SEEurope-Network 2007; Gold/Schwimbersky 2007), wobei drei dominieren:

- „Leere“ SEs verfügen über wirtschaftliche Aktivitäten (z. B. halten sie Vermögen), jedoch haben sie keine Beschäftigten.

- „Vorrats“-SEs (Blanke 2005) weisen weder das eine noch das andere Merkmal auf. - Von sogenannten „UFO“-SEs (Schwimbersky 2006) ist nur ihre Existenz durch die Eintragung im Handelsregister bekannt. Mit großer Wahrscheinlichkeit handelt es sich ebenfalls um "leere" bzw. "Vorrats“SEs, eine Häufung von Begriffen wie „Capital“, „Finance“ oder „Invest“ könnte darauf hindeuten, dass es möglicherweise ökonomische Aktivitäten gibt, aber keine Arbeitnehmer.

Für unsere Analyse aus einer IndustrialRelations-Perspektive sind diese Subtypen nicht von direktem Interesse. Es ist noch keine eindeutige Aussage über deren Gründungszweck möglich; es wäre z. B. ein Verkauf an ein anderes Unternehmen denkbar, um dessen Gründungsprozess zu vereinfachen bzw. zu beschleunigen. Erst wenn in einem solchen Fall Arbeitnehmer betroffen wären, könnte eine gewisse Relevanz für die hier eingenommene Perspektive entstehen.

\section{2 "NORMALE" SES}

Im Gegensatz zu den dargestellten Subtypen beruht unsere folgende Analyse auf „normalen“ SEs, also Unternehmen, die wirtschaftliche Aktivitäten und Arbeitnehmer aufweisen. Mitte 2007 sind 19 solcher SEs in die nationalen Handelsregister eingetragen, ca. fünf weitere sind im Prozess der Gründung. Nur rund $15 \%$ der registrierten SEs sind also normale Unternehmen (Übersicht 1).

Die meisten dieser 24 SEs werden auf dem Wege der Umwandlung gegründet (20 Fälle); vier werden mittels einer Verschmelzung errichtet. Die beiden Gründungsformen einer Holding- bzw. Tochter-SE spielen bei den „normalen“ Fällen bisher keine Rolle.

Untersucht man diese Unternehmen hinsichtlich der Verteilung nach Unternehmensgröße oder -sektor, sind keine eindeutigen Tendenzen festzumachen. Ihre Größe schwankt beträchtlich; kleinere und mittlere sind ebenso vertreten wie „Weltkonzerne" mit 100.000 und mehr Arbeitnehmern. In zeitlicher Hinsicht sind ebenfalls noch keine klaren Trends festzustellen. Die Anzahl gegründeter SEs hat sich im Jahre 2006 (fünf gegründete „normale“ SEs) im Vergleich zu 2005 (vier Gründungen) nicht deutlich verändert.

Auch die Tatsache, dass einige Wirtschaftssektoren im Hinblick auf die Verteilung ihrer Produktionsstätten und ihrer Arbeitnehmerschaft internationalisierter sind als andere, spiegelt sich (noch) nicht in einer höheren Anzahl von SEs wider. Die bestehenden SEs finden sich sowohl im Produktions- als auch im Dienstleistungssektor. Die Entscheidung zur Gründung einer SE scheint mit unternehmensspezifischen Motiven zusammenzuhängen, wie der Vereinfachung von Strukturen und damit verbundene Kosteneinsparungen und nicht mit sektorspezifischen Merkmalen.

Bei der Verteilung nach dem Sitzland stellt man fest, dass die meisten „,normalen“ SEs nur in einer begrenzten Anzahl von Ländern registriert werden. Zwölf Unternehmen haben ihren Sitz in Deutschland, drei in Österreich, außerdem sind vor allem die skandinavischen Länder betroffen. Nur zwei „normale“ SEs haben ihren Sitz in neuen EU-Mitgliedstaaten (Übersicht 1). Für die SE spielte die sogenannte „EUOsterweiterung “ im Gegensatz zur EBR-RL - zumindest bisher - keine nennenswerte Rolle. Ein Grund könnte sein, dass die SE lediglich eine zusätzliche Option für Unternehmensleitungen darstellt, während es im Falle der EBR-RL eine Pflicht zur Einrichtung gibt, sofern eine der beiden Seiten im Unternehmen aktiv wird. Unternehmen mit Konzernsitz in nicht-europäischen Ländern sind bisher nicht an Gründungen „normaler" SEs im hier verwandten Sinne beteiligt.

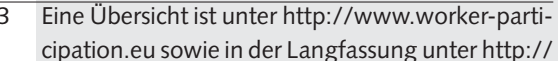
cipation.eu sowie in der Langfassung unter http://
www.sozialmodell.eu (Fußnote 1) erhältlich. 


\begin{tabular}{|c|c|c|c|}
\hline $\begin{array}{l}\text { SE (Name) } \\
\text { AN-Zahl weltweit }\end{array}$ & $\begin{array}{l}\text { Beteiligte Länder mit AN-Zahl, sofern verfügbar } \\
\text { (Sitzland fett) }\end{array}$ & Branche & $\begin{array}{l}\text { Datum d. } \\
\text { Eintragung }\end{array}$ \\
\hline Alfred Berg SE [322 AN] & DK (59); FIN (66); N (53); S (144) & Bank & 30.09 .05 \\
\hline Allianz SE [162.000 AN] & $\begin{array}{l}\text { B (1.955); DK; D (87.986); EST; FIN; F (20.779); GR (562); IRL (1.106); } \\
\text { I (7.537); LV; LT; L; NL (2.170); N; PL (400); P (891); AT (3.130) S (112); } \\
\text { SK; E (2.783); CZ (937); H (3.000); GB (8.688); CY }\end{array}$ & $\begin{array}{l}\text { Versicherung/ } \\
\text { Allfinanzgruppe }\end{array}$ & 13.10 .06 \\
\hline BASF AG [95.247 AN] & $\begin{array}{l}\text { B (4.101); BG (17); DK (297); D (51.148); FIN (128); F (2.352); GR (79); } \\
\text { IRL (16); I (1.517); LT (16); M (1); NL (1.096); N (32); AT (119); PL (271); } \\
\text { P (450); RO (32); S (198); SK (130); SLO (38); E (1.919); CZ (230); H (132); } \\
\text { GB (1.271) }\end{array}$ & Chemie & Verhandlungen laufen \\
\hline Conrad Electronic SE [2.314 AN] & D (2.313); AT (1) & Handel & 18.08 .06 \\
\hline Donata Holding SE [3.922 AN] & D (564); E (936); F (786); GB (745); PL (232) u. 10 weitere Länder & $\begin{array}{l}\text { Kosmetik-/ } \\
\text { Parfümindustrie }\end{array}$ & $\begin{array}{l}21.03 .06 \\
\text { (als Vorrats-SE) }\end{array}$ \\
\hline Elcoteq SE [19.600 AN] & $\begin{array}{l}\text { EST (3.342); D (548); FIN (861); S (7); H (2.692) } \\
\text { Sitzverlegung nach L angekündigt }\end{array}$ & Elektroindustrie & 01.10 .05 \\
\hline $\begin{array}{l}\text { Fresenius SE [45.777 AN } \\
\text { im EWR] }\end{array}$ & $\begin{array}{l}\text { B (108); DK (16); D (29.288); EST (24); FIN (17); F (2.269); GR (37); } \\
\text { IRL (7); I (1.223); L (11); NL (709); N (403); AT (2.301); PL (2.040); } \\
\text { P (1.037); S (889); SK (348); SLO (100); E (1.808); CZ (1.397); H (770); } \\
\text { GB (975) }\end{array}$ & $\begin{array}{l}\text { Medizinische } \\
\text { Dienstleistungen } \\
\text { und Chemie }\end{array}$ & 13.07 .07 \\
\hline $\begin{array}{l}\text { Galleria di base del Brennero SE } \\
\text { [33 AN] }\end{array}$ & I; AT & Bauindustrie & 17.12 .04 \\
\hline Graphisoft SE [253 AN] & $\begin{array}{l}\mathrm{D} ; \mathrm{NL} ; \mathrm{E} ; \mathrm{H} ; \mathrm{GB} \\
\text { Sitz vormals in NL }\end{array}$ & IT & 27.07 .05 \\
\hline Hager SE [ca. 2.000 AN] & $\mathrm{D} ; \mathrm{F}$ & Metallindustrie & 15.06 .07 \\
\hline Lyreco CE SE [49 AN] & AT (9), SK (30), CZ (5), H (5) & Handel/Vertrieb & 08.10 .05 \\
\hline MAN Diesel SE [6.700 AN] & DK; D; F; GR; NL; S; E; CZ; GB & Metallindustrie & 01.09 .06 \\
\hline $\begin{array}{l}\text { Mensch und Maschine SE } \\
\text { [350 AN] }\end{array}$ & $\mathrm{B} ; \mathrm{D} ; \mathrm{F} ; \mathrm{I} ; \mathrm{AT} ; \mathrm{PL} ; \mathrm{S} ; \mathrm{GB}$ & IT & 07.12 .06 \\
\hline Nordea Bank [29.000 AN] & B; DK; D; EST; FIN; F; LV; LT; L; N; PL; E; S; GB & Bank & $\begin{array}{l}\text { Verhandlungen } \\
\text { abgeschlossen / } \\
\text { Eintragung stockt wg. } \\
\text { Problemen mit der } \\
\text { Einlagensicherung }\end{array}$ \\
\hline Odfjell SE [3.500 AN] & $\mathrm{NL} ; \mathbf{N} ; \mathrm{GB}$ & Schifffahrt & 23.07 .07 \\
\hline PCC SE [3.756 AN] & D (136); FIN (3); LV; PL (3.581); SK (3); CZ (33) & Chemie & 05.02 .07 \\
\hline Plansee SE [2.200 AN] & F (76); AT (1.333); S (2); GB (11) & Metallindustrie & 11.02 .06 \\
\hline Prosafe SE [55 AN im EWR] & N (10); GB (45) & Ölindustrie & 17.11 .06 \\
\hline $\begin{array}{l}\text { Porsche Holding } \\
\text { [ca. 11.500 AN] }\end{array}$ & B; D; u.a. & Automobilindustrie & $\begin{array}{l}\text { Verhandlungen } \\
\text { abgeschlossen; } \\
\text { Klage des } \\
\text { VW-Betriebsrates } \\
\text { gegen Eintragung }\end{array}$ \\
\hline Riga RE - SE [28 AN] & $\mathrm{D} ; \mathrm{I} ; \mathrm{LV} ; \mathrm{LT} ; \mathrm{GB}$ & Rückversicherung & 11.08 .06 \\
\hline Sevic Systems SE [ca. 100 AN] & D u. a. & IT & 15.03 .07 \\
\hline $\begin{array}{l}\text { Strabag Bauholding SE } \\
\text { [31.000 AN] }\end{array}$ & $\begin{array}{l}\text { B; D (10.177); I; NL (202); AT (11.227); PL (1.055); SK; SLO; CZ (2.801); } \\
\text { H (4.352) }\end{array}$ & Bau & $\begin{array}{l}\text { 12.10.04, } \\
\text { Verhandlungen } \\
\text { nachträglich (!) } \\
\text { abgeschlossen } \\
(04.05 .06)\end{array}$ \\
\hline $\begin{array}{l}\text { SCOR SE/SCOR Global P\&C } \\
\text { SE/SCOR Global Life SE } \\
{[1.211 \text { AN] }}\end{array}$ & F und 17 weitere Länder & Rückversicherung & 25.06 .07 \\
\hline Surteco SE [2.109 AN] & D; I; PL; GB & $\begin{array}{l}\text { Papier- und } \\
\text { Kunststoffindustrie }\end{array}$ & $\begin{array}{l}\text { Verhandlungen } \\
\text { abgeschlossen, } \\
\text { Eintragung steht bevor }\end{array}$ \\
\hline
\end{tabular}

\section{Arbeitnehmerbeteiligung
in der SE}

\subsection{GRUNDPRINZIPIEN}

Die Grundsatzentscheidung zur SE-Gründung fällen die Leitungsorgane ohne Interventionsmöglichkeit der Arbeitnehmervertreter. Die Unternehmensleitung stellt einen „Gründungsplan“ auf, der unter an- derem über die Form der SE entscheidet. Auch die Organstruktur wird festgelegt, wobei zwei „Grundtypen“ infrage kommen: Einerseits eine monistische Organstruktur, wie im Vereinigten Königreich üblich, d.h. ein Verwaltungsorgan (board) führt das Unternehmen; andererseits eine dualistische Organstruktur, d.h. die Separierung von Unternehmensleitung und -kontrolle in zwei Organe (Vorstand und Aufsichtsrat). Daneben sind auch „kombinierte Systeme" denkbar, wie in Frankreich (Keller 2002; zu einer Übersicht nach Län- dern: Kluge/Stollt 2006). Mit der Rechtsform der SE wird es Unternehmen erstmals möglich, in einem Staat, in dem bisher nur eine dualistische Form zugelassen war, nunmehr auch eine SE mit monistischer Struktur zu gründen - und umgekehrt.

Nach Aufstellung dieses Gründungsplans muss die Unternehmensleitung Verhandlungen zur Beteiligung der Arbeitnehmer einleiten, mit dem Ziel, eine Vereinbarung abzuschließen. Ohne Verhandlungen ist eine Eintragung der SE in das nationale 
Handelsregister grundsätzlich nicht möglich (Art. 12 SE-VO). Insofern wird einer einseitigen Entscheidung des Managements zur Arbeitnehmerbeteiligung vorgebeugt. Gerade Arbeitnehmervertretern aus EWR-Mitgliedstaaten mit ausschließlich freiwilliger Mitbestimmungstradition (van het Kaar 2005) bietet die SE zum ersten Mal die Gelegenheit, unternehmerische Entscheidungsprozesse auf Konzernebene mitzugestalten (van het Kaar 2006).

Für Umfang und Inhalte der Arbeitnehmerbeteiligung macht die Richtlinie, wie erwähnt, keine detaillierten inhaltlichmateriellen Vorgaben.

Alle Fragen zur Arbeitnehmerbeteiligung sind also frei verhandelbar mit grundsätzlich offenem Ausgang. Die Vereinbarung soll mindestens Verabredungen enthalten zur Unterrichtung und Anhörung der Arbeitnehmer in einem Vertretungsorgan oder „SE-Betriebsrat“. Art. 4 der SE-RL sieht vor, dass eine Beteiligungsvereinbarung auch Fragen der Unternehmensmitbestimmung regeln kann, z. B. die „Zahl der Mitglieder des Verwaltungs- oder Aufsichtsorgans der SE, welche die Arbeitnehmer wählen [...] und die Rechte dieser Mitglieder". Es wird also üblicherweise um zwei Ebenen von Arbeitnehmerbeteiligung verhandelt.

\subsection{DAS BESONDERE VERHANDLUNGSGREMIUM}

In der Verhandlungsphase repräsentiert ein „besonderes Verhandlungsgremium“ (BVG) die Arbeitnehmer; auf Arbeitgeberseite führt der Vorstand die Verhandlungen. Die Mitglieder des BVG werden gemäß Art. 3 der SE-RL gewählt, vereinfacht ausgedrückt: proportional zu ihrer Anzahl in den betroffenen Mitgliedstaaten, je nach Land unterschiedlich entweder von den nationalen Betriebsräten, den jeweiligen Gewerkschaften oder direkt von der Belegschaft (Fulton 2006). In Unternehmen, in denen bereits ein EBR bestand, spiegelt die Zusammensetzung des BVG in der Regel die des EBR wider. ${ }^{4}$ Diese Konstruktion trägt dazu bei, dass die Kooperation zwischen den einzelnen Mitgliedern des BVG sowie der Austausch von Informationen leichter vonstatten geht, da auf eine bereits existierende Form institutionalisierter Zusammenarbeit zurückgegriffen werden kann. Die Tatsache, dass erfahrene Arbeitnehmervertreter beteiligt sind, wirkt positiv auf die inhaltliche Kompetenz.
Einige Mitglieder des BVG, insbesondere der Vorsitzende sowie die Repräsentanten der Länder, in denen die meisten Arbeitnehmer beschäftigt sind, spielen eine wichtigere Rolle für Verlauf und Ausgang der Verhandlungen als andere. Diese Mitglieder verabreden sich informell zwischen den Sitzungen; häufig gehören sie auch kleineren "Verhandlungsteams “ an, die gebildet werden, wenn das BVG sehr viele Mitglieder aufweist (z. T. über 30), sodass ein effizientes Verhandeln des "großen“ BVG mit dem Management nicht möglich wäre. Das BVG überträgt das Verhandlungsmandat entweder komplett oder teilweise auf das kleinere Team; regelmäßige Berichts- sowie eine Zustimmungspflicht sind vorgesehen. Die Mitglieder des BVG sind in aller Regel in der zuständigen Gewerkschaft organisiert. Es gab nur sehr wenige Ausnahmen, in denen einzelne Belegschaften Unorganisierte ins BVG entsandt haben, z. B. leitende Angestellte.

\subsection{EXTERNE RESSOURCEN}

Beide Seiten können externe Experten zu den Verhandlungen hinzuziehen (Art. 3 (5) SE-RL). Die Arbeitgeberseite beteiligt häufig Rechtsanwaltskanzleien als Berater. Diese Strategie verursacht typische PrinzipalAgenten-Probleme: Ziel der Anwaltskanzleien kann sein, eine Vereinbarung zu erzielen, die sich als „Werbung“ für neue Fälle einsetzen lässt. Dabei besteht die Gefahr, dass diese Berater durchaus auch Konzessionen zuungunsten aller spezifischen Belange des betroffenen Unternehmens und seiner Arbeitnehmer eingehen.

Die Kosten für Sachverständige des BVG sind vom Unternehmen zu tragen, wobei nach den meisten Umsetzungsgesetzen nur ein Sachverständiger vorgesehen ist (EWCs Bulletin 2006). Die nationalen Regelungen können vorsehen, dass Gewerkschaftsvertreter dem BVG - nicht nur als Sachverständige - auch dann direkt angehören, wenn sie nicht Arbeitnehmer eines der beteiligten Unternehmen sind. In der Regel spielt die zuständige nationale Gewerkschaft eine besondere Rolle bei der Unterstützung der Arbeit des BVG, auch wenn meistens eine Rückkopplung zu den europäischen Gewerkschaftsförderationen gegeben ist. Gerade europäische Gewerkschaftsvertreter können zu einer Balance zwischen unternehmensegoistischen und eher generellen Arbeitnehmerinteressen beitragen. Strategien des Managements,
Gewerkschaften möglichst außen vor zu halten, dürften hier weniger Erfolg versprechend und weniger verbreitet sein.

Die Gewerkschaftsföderationen spielen eine weitere Rolle: Wie bereits im Falle der EBR erstellen sie für die Verhandlungen Richtlinien und Handreichungen (z. B. EMF 2003). Die SE ist eine weitere Herausforderung, die die Fähigkeiten der Gewerkschaften zu supranationaler Koordinierung fordert-und Ressourcen verlangt wie auch bindet.

\subsection{VERHANDLUNGSSZENARIEN}

Die eigentlichen Verhandlungen zwischen BVG und Unternehmensleitung dauern sechs Monate und können bis zu einem Jahr verlängert werden (Art. 5 SE-RL). Drei Verhandlungsergebnisse sind grundsätzlich denkbar (Köstler 2006):

(1) Der „Regelfall“ ist der Abschluss einer Vereinbarung zur Beteiligung der Arbeitnehmer nach Art. 4 SE-RL. Diese Vereinbarung erfolgt in „unbeschadet [er...] Autonomie der Parteien“ im Hinblick auf Inhalte; jedoch gilt im Falle der Gründung durch Umwandlung, dass alle Komponenten und das Ausmaß der Arbeitnehmerbeteiligung Bestandsschutz haben müssen.

Eine Vereinbarung regelt im Normalfall beide genannten Ebenen. Bei der Unterrichtung und Anhörung der Arbeitnehmer werden Regelungen getroffen zum Vertretungsorgan/SE-Betriebsrat (z. B. Zusammensetzung, Befugnisse und Rechte zur Information, Konsultation und ggf. Mitentscheidung, Sitzungshäufigkeit, Arbeitsausstattung). Bei der Arbeitnehmerbeteiligung in den Leitungsorganen wird z. B. um die Zahl der Mitglieder im Aufsichtsrat (AR) oder im board verhandelt.

(2) Statt einer Vereinbarung können die in Art. 7 (1) SE-RL vorgesehenen Auffangregelungen greifen. Dies wäre der Fall, wenn beide Parteien bis zum Ende des Verhandlungszeitraums keine Vereinbarung erzielen bzw. die Anwendung der Auffangregelungen beschließen. Diese bestehen aus drei Teilen, nämlich Regelungen zum Ver-

\footnotetext{
4 Das BVG wird immer neu errichtet. Häufig gewählt werden jedoch Arbeitnehmer, die Mitglied eines bereits existierenden EBRs sind; ein EBR selbst kann nie als BVG fungieren.
} 
tretungsorgan, zu Unterrichtung und Anhörung sowie in bestimmten Fällen zu Mitbestimmung in den Leitungsorganen.

(3) Schließlich ist eine „Nulllösung“ denkbar, wenn das BVG keine Verhandlungen mit der Unternehmensleitung aufnimmt oder diese abbricht. Für die Beteiligung der Arbeitnehmer gelten dann weiterhin die nationalen Regelungen, zusätzlich findet ggf. die EBR-RL Anwendung. ${ }^{5}$

Aus verhandlungstheoretischer Perspektive stellt das unter Punkt 2 genannte Szenario die Option dar, die mit Blick auf die Erfahrungen der EBR in der „Art. 6-Phase“ als „Mindeststandard“ bezeichnet wird. Die Verhandlungen dürften sich um diese Mindeststandards bewegen. In empirischer Perspektive wurde bisher in jedem Fall eine Vereinbarung abgeschlossen, sieht man von den beiden Fällen mit Sitz in neuen EU-Mitgliedstaaten ab. ${ }^{6}$ Größtenteils spiegeln die Vereinbarungen, wie die Abschnitte 4 und 5 detailliert belegen, die Inhalte wider, welche die Auffangregelung vorgesehen hätte, auch wenn gelegentlich „tradeoffs" erzielt wurden.

\subsection{VERHANDLUNGSPROZESSE INNERHALB DES BVG}

Empirische Untersuchungen zu den Verhandlungen bei der EBR-Einrichtung (Lecher et al. 2002) zeigen, dass Verhandlungsprozesse nicht nur zwischen Unternehmensleitung und BVG stattfinden, sondern auch innerhalb des BVG, sodass wir es neben einem inter- auch mit einem intraorganisationellen Verhandlungsprozess (Walton/McKersie 1991) zu tun haben. Arbeitnehmervertreter aus verschiedenen Mitgliedstaaten bringen in das BVG ihre nationalen Erfahrungen ein, die auf verschiedenen rechtlichen und institutionellen Arrangements aufbauen. Gewerkschaftsvertreter als externe Experten versuchten unterschiedliche Vorstellungen im BVG zu balancieren. Einzelne BVG-Vertreter kannten andere nationale Traditionen oft nicht, hier konnten bereits Informationen zu einem Interessensausgleich beitragen.

Konflikte innerhalb des BVG traten häufig auch auf in Fragen wie Arbeitssprache und Simultanübersetzungen, aber auch bei der Sitzverteilung in den Gremien der SE, insbesondere im Aufsichtsrat. Wiederum fungierten Gewerkschaftsvertreter als Mediatoren, oft gelangen Lösungen durch
Verweise auf die rechtliche Situation. Solche internen Schwierigkeiten und Konflikte müssen gelöst und gemeinsame Positionen gefunden werden, bevor die eigentlichen Verhandlungen beginnen. Ansonsten würden solche Konflikte die eigene Verhandlungsposition schwächen. Trotzdem zeigen die bisherigen Fälle, dass entsprechende Übereinkünfte schwierig waren, zum Teil gelangen sie erst während der Verhandlungen.

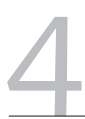

\section{SE-Betriebsrat}

Die Vertretungsorgane zur Unterrichtung und Anhörung der Arbeitnehmer, die sogenannten „SE-Betriebsräte“ (SE-BR), reflektieren oft vorher existierende EBRs im Hinblick auf Mitglieder und Strukturen. Hinsichtlich der Arbeitsteilung zwischen SE-BR und den nationalen Gremien wurde vielfach ein Passus zu Kompetenzabgrenzungen aufgenommen: Der SE-BR ist für die Angelegenheiten zuständig, die die SE selbst bzw. Arbeitnehmer in mehreren Mitgliedstaaten betreffen, während die nationalen Gremien weiterhin zuständig sind für alle Fragen, die lediglich nationale Arbeitnehmer bzw. einen nationalen Unternehmensteil anbelangen. Oft regeln die Vereinbarungen, dass die nationalen Gremien bestimmte Aufgaben auf den SE-BR übertragen können, solange nationale rechtliche Anforderungen nicht berührt werden. ${ }^{7}$

Die Rechte des SE-BR sind in allen vorliegenden Vereinbarungen beschränkt auf Unterrichtung und Anhörung. Verhandlungs- im Sinne echter Mitentscheidungsrechte sind nicht vorgesehen. Auch wenn die Rechte der SE-BR damit die der vorher existierenden EBRs nicht wesentlich überschreiten, gibt es in manchen Fällen aus Arbeitnehmersicht Verbesserungen. Diese betreffen vor allem technisch-institutionelle Fragen wie eine verbesserte Institutionalisierung der Treffen mit dem Management, bestimmte Initiativrechte oder die Einführung eines „Controlling“-Systems zur Evaluation des Zusammenwirkens beider Seiten.

\subsection{GRÖßE UND ZUSAMMEN- SETZUNG}

Die Größe des SE-BR berechnet sich in der Regel nach der Anzahl der Arbeitnehmer in der SE bzw. nach ihrer nationalen Verteilung. Oft spiegelt seine Zusammensetzung die des BVG wider, sowohl hinsichtlich der Verteilung der Sitze nach Ländern als auch hinsichtlich der konkreten Personen. In den meisten Fällen war die Frage nach der Sitzverteilung im SE-BR kein wesentlicher Verhandlungsgegenstand innerhalb des BVG.

Betrachtet man wieder die Parallelen zu den EBR, stellt sich die Frage, ob die SEBR eher nach dem deutschen Modell nur Arbeitnehmervertreter als Mitglieder haben, oder ob es sich eher, dem französischen Modell folgend, um sogenannte „joint bodies“ handelt. Die SE-BRs bestehen in allen Beteiligungsvereinbarungen nur aus Arbeitnehmervertretern, lediglich in einer SE mit Sitz in Frankreich wird ein „joint body" eingerichtet. Die SE-BR folgen also nationalen Pfadabhängigkeiten.

Kerckhofs (2006) stellt fest, dass bei der Mehrzahl der EBRs kleinere Steuerungsgremien, die meist aus dem Vorsitzenden und seinen Stellvertretern bestehen, für die tägliche Arbeit existieren. Eine Auswertung für SE-BRs zeigt ein ähnliches Bild, vor allem für die größeren SEs. Für die Praxis ist anzunehmen, dass bestimmte Mitglieder eine solche Funktion auch auf informeller Basis übernehmen werden, ohne dass die Vereinbarung dies explizit regelt.

\subsection{RESSOURCEN}

Die Ausstattung mit Ressourcen bestimmt die Optionen und Restriktionen eines SEBR. Die Frage nach Freistellungen spielt eine ebenso entscheidende Rolle wie die nach Schutz vor Diskriminierung durch den

\footnotetext{
5 Interessanterweise war die heute mögliche "Nulllösung " in den Vorschlägen der "Davignon"Kommission nicht vorgesehen: Diese schlug eine Sockellösung von zwei Arbeitnehmersitzen in den Leitungsorganen vor.

6 Hierbei handelt es sich um kleine Unternehmen, über die Informationen nur sehr spärlich verfügbar sind: Im Falle der Lyreco CE SE wurde zwar ein BVG einberufen; es beschloss jedoch die Anwendung der Nulllösung. Im Falle der Riga RE SE wurde, soweit dazu Informationen verfügbar sind, kein BVG einberufen. Es ist insofern fraglich, ob diese SE überhaupt ins lettische Handelsregister hätte eingetragen werden dürfen.

7 Art. 4 SE-RL erlaubt statt der Einrichtung eines Vertretungsorgans, also eines SE-BR, auch die Einrichtung eines anderen Verfahrens zur Unterrichtung und Anhörung der Arbeitnehmer; ähnlich wie im Falle der EBR spielt diese Möglichkeit auch im Falle der SE keine Rolle.
} 
Arbeitgeber, z. B. Kündigungsschutz. Die Vereinbarungen verweisen häufig auf die nationalen Bestimmungen, die für den jeweiligen Arbeitnehmer gelten. Gerade Kündigungsschutzregelungen können insofern für verschiedene Mitglieder des SEBR unterschiedlich ausfallen.

Die Mehrzahl der Vereinbarungen sieht jährlich zwei ordentliche Zusammenkünfte mit der Unternehmensleitung vor; bisweilen konnten sich die Parteien nur auf ein Treffen einigen. Außerordentliche $\mathrm{Zu}$ sammenkünfte sind dann möglich, wenn "essentielle“ oder „unvorhersehbare“ Ereignisse eintreten, die die Arbeitnehmer betreffen. Vorbereitende Treffen des SE-BR sind ebenfalls vereinbart. Alle Kosten für diese Treffen, insbesondere für Übersetzungen, trägt das Unternehmen.

In Bezug auf Fort- und Weiterbildung für die Mitglieder des SE-BR konnten die Arbeitnehmervertreter in den meisten Vereinbarungen eine relativ große Autonomie erreichen. Bestimmte inhaltliche Themengebiete sind in den Vereinbarungen nicht vorgegeben, die Maßnahmen müssen lediglich der „Arbeit des SE Betriebsrats erforderliche Kenntnisse vermitteln“ (Köstler 2007, S. 66). In den meisten Fällen versuchten die Unternehmensleitungen, Englisch als Arbeitssprache zu installieren, jedoch akzeptierten sie auch, weiterhin Kosten für Dolmetscher zu tragen. Auch die Kosten für externe Sachverständige, die der SE-BR hinzuziehen kann, übernehmen nach den meisten Vereinbarungen die Unternehmen.

Einige Gemeinsamkeiten, aber auch Unterschiede, betreffen die Modalitäten bezüglich Laufzeit und Kündigungsmöglichkeiten der ersten Beteiligungsvereinbarung. In den meisten Fällen wurde eine relativ lange Laufzeit vereinbart, von vier bis zu zehn Jahren. ${ }^{8}$ In manchen Fällen können Teile der Vereinbarung (zum SE-BR bzw. zur Mitbestimmung in den Leitungsorganen) auch getrennt gekündigt werden. Andere Vereinbarungen sehen eine Kündigungsmöglichkeit dagegen nur in Gänze vor. Die Kündigungsfristen divergieren zwischen sechs Monaten und einem Jahr.

\subsection{DIE BEDEUTUNG DER AUFFANGREGELUNGEN}

Nach Art. 7 SE-RL bestehen bestimmte Auffangregelungen zur Unterrichtung und Anhörung der Arbeitnehmer für den Fall, dass die Verhandlungen scheitern. Wie dar- gelegt, ist anzunehmen, dass die Verhandlungsprozesse häufig um diese „Mindeststandards" kreisen, die Inhalt und Umfang der Beteiligungsvereinbarung vordefinieren. Diese Auffangregelungen stellen einen "Schatten des Gesetzes" (Bercusson 1992) dar. In der Logik von rational choice kann die eine Seite keine niedrigeren Standards anbieten, zumindest dann nicht, wenn das komplette Scheitern der Verhandlungen ausgeschlossen werden soll. Damit verbunden wäre eine erhebliche Verschlechterung der Beziehungen beider Seiten, die weiterhin kooperieren müssen. Andererseits weiß der Verhandlungspartner um die Schwierigkeit, eine weitergehende Vereinbarung $\mathrm{zu}$ erreichen als von den „Mindeststandards" vorgesehen. Vor diesem Hintergrund überrascht nicht, dass sich die Vereinbarungen in der Tat um die in den Auffangregelungen vorgegebenen „Mindeststandards" bewegen.

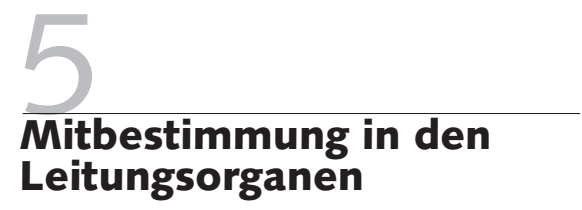

In den Mitgliedstaaten existieren verschiedene Formen der Corporate Governance. Es gibt monistische Organstruktursysteme, dualistische sowie Mischformen; 19 von 27 EU-Mitgliedstaaten sehen Formen von Arbeitnehmerbeteiligung in den Leitungsorganen vor (Kluge/Stollt 2007). In quantitativer wie vor allem in qualitativer Hinsicht gibt es erhebliche Unterschiede. Eine Evaluation der Effizienz der verschiedenen Systeme ist schwer möglich. Nach Fällen wie Enron oder Worldcom in den USA steht fest, dass eine monistische Organstruktur einer dualistischen nicht automatisch überlegen ist, wie in den 1990er Jahren wiederholt behauptet wurde (Nagel 2006).

\subsection{CORPORATE GOVERNANCE IN DEN ERSTEN SES}

Wie erwähnt, können Unternehmen, die eine SE gründen, mit dieser neuen Rechtsform zum ersten Mal frei entscheiden, welche Organstruktur sie implementieren. Auch spätere Wechsel sind grundsätzlich möglich. Eine Analyse der "normalen“ SEs zeigt, dass beide Systeme vorkommen: 13 Fälle weisen eine monistische, neun eine dualistische Organstruktur auf. In der Mehrzahl der Fälle spiegelt die Organ- struktur diejenige wider, die das Unternehmen vor der Errichtung der SE aufwies und die das Sitzland kennzeichnet. Dieser Sachverhalt ist nicht überraschend, solche organisationelle Kontinuität oder Pfadabhängigkeit wurde mehrfach nachgewiesen (Villiers 2006).

Immerhin gibt es sechs Ausnahmen. Der Wechsel fand jeweils vom dualistischen ins monistische System statt, einen Wechsel in umgekehrter Richtung gab es noch nicht. Der gemeinsame Nenner besteht darin, dass es sich um eigentümerzentrierte Unternehmen handelt, bei denen der Eigentümer stark in das Tagesgeschäft involviert ist. ${ }^{9}$ Eine wie auch immer geartete Kooperation mit einem Aufsichtsrat (AR) wird als unnötig angesehen, eine Teilung der Leitungsorgane würde nur Strukturen verteuern und Prozesse verlangsamen, so die Stellungnahmen von Beteiligten.

\subsection{GRÖSSE UND ZUSAMMEN- SETZUNG}

Die Grundsatzentscheidung über die Größe der Leitungsorgane treffen die Eigentümer der SE in Zusammenarbeit mit dem Vorstand, z. B. auf der Hauptversammlung. Diese Entscheidung fällt bereits mit dem Gründungsplan, also bevor Verhandlungen mit dem BVG stattfinden. Jedoch muss die von der Hauptversammlung beschlossene Satzung angepasst werden, falls die Verhandlungen zur Arbeitnehmerbeteiligung eine andere Größe des AR ergeben.

Mit der SE-Gründung ging im Falle dualistisch strukturierter Unternehmen mehrfach eine Verkleinerung des AR einher, d.h. die gleiche Zahl an Mitgliedern sowohl auf Arbeitgeber- wie auf Arbeitnehmerseite wurde „eingespart“. Die Unternehmensleitungen setzen immer die Agenda für die Zusammensetzung der Leitungsgremien. Auch wenn das BVG bzw. einzelne Mitglieder versuchen, dieses The-

\footnotetext{
8 Bei einer aus Arbeitnehmersicht besonders unvorteilhaften Vereinbarung werden sogar keinerlei Aussagen zu einer Kündigungsmöglichkeit gemacht.

9 Lyreco CE SE mit Sitz in SK gehört zur französischen Lyreco-Gruppe. Hier könnte sich die monistische Struktur zum einen durch die geringe Größe erklären, zum anderen durch die monistische Organstruktur im Sitzland des Mutterkonzerns. Man kann hier von einer Pfadabhängigkeit über die nationalen Grenzen hinweg sprechen.
} 
ma in den Verhandlungen zur Sprache zu bringen, wird aus Arbeitnehmersicht praktisch nichts erreicht. Einerseits wurde zwar in keinem Fall die „paritätische“ Besetzung der Gremien eingeschränkt, sofern es diese im Vorfeld gab; dies wäre auch durch die gesetzlichen Auffangregelungen kaum möglich gewesen. Die Machtressourcen der BVGs reichten nicht aus, um eine Reduzierung der Größe der Gremien oder gar einen Wechsel vom dualistischen ins monistische System zu verhindern.

In allen SEs haben sämtliche Mitglieder der Leitungsorgane zumindest formal die gleichen Rechte. Dennoch gibt es Aussagen, dass es für Arbeitnehmervertreter bisweilen eine Herausforderung darstelle (Schwimbersky/Rehfeldt 2006, S. 15), vollständig in die Arbeit des board eingebunden $\mathrm{zu}$ werden und alle Informationen $\mathrm{zu}$ erhalten, welche anderen Mitgliedern zur Verfügung stehen.

\subsection{VERHÄLTNIS SE-BR UND UNTERNEHMENSMITBESTIMMUNG}

Aus juristischer Perspektive sind die beiden Ebenen der Beteiligung unabhängig voneinander. Einige Beobachter erwarteten, dass sich die Verhandlungen vor allem um die Unternehmensmitbestimmung drehen würden. Die empirische Analyse zeigt, dass das nicht der Fall ist. Beide Ebenen sind faktisch nicht unabhängig. Tradeoffs in verschiedener Hinsicht sind zu konstatieren: Zum einen betreffen sie interne Interessen auf Arbeitnehmerseite, wie die Verteilung von Sitzen. Bei einer getrennten Betrachtung der BVG-Mitglieder als betriebliche bzw. als externe Gewerkschaftsvertreter fällt auf, dass Erstere mit der Arbeitgeberseite eher Fragen des SE-BR, Letztere eher Fragen der Unternehmensmitbestimmung thematisierten. Zwischen beiden Gruppen gibt es dennoch vielfältige Interaktionen. Gewerkschaftsvertreter fungieren als Informationsquellen für die betrieblichen BVG-Mitglieder; sie unterstützen diese bei inhaltlichen Fragen und organisieren eine Koordination der Akteure innerhalb des BVG.

Zum anderen sind trade-offs auch bei den Ergebnissen der Verhandlungen zwischen BVG und Unternehmensleitung festzustellen. Mussten die Mitglieder des BVG Konzessionen eingehen, wie im Hinblick auf die Größe der Leitungsorgane, griffen sie dieses Argument auf, um mehr Rechte und eine bessere Ressourcenausstattung für den SE-BR zu erreichen (z. B. in Fragen der Zugänglichkeit des Managements für den SE-BR). Hier kann man Anzeichen eines „do ut des“ Verhandlungsprozesses feststellen, der dem Prinzip von Geben und Nehmen folgt. Im Vergleich mit EBRs sind die Möglichkeiten für solche trade-offs umfassender aufgrund der beiden Ebenen, um die es in den Verhandlungen bei der Gründung einer SE geht.

Nationale Traditionen können im Verhältnis der Verhandlungen zu beiden Ebenen wiederum eine Rolle spielen. Die Arbeitnehmervertreter eines Landes, in dem Arbeitnehmerbeteiligung in den Leitungsorganen unbekannt ist, werden an diesem Themenkomplex weniger Interesse zeigen und stattdessen für den SE-BR eintreten. In den meisten Fällen dualistisch strukturierter SEs sind Mitglieder des SE-BR auch Arbeitnehmervertreter in den Leitungsorganen. Dieses Arrangement kann zu engen und lebendigen Arbeitsbeziehungen im Unternehmen beitragen.

\subsection{EINIGE ÜBERLEGUNGEN ZU DEN DEUTSCHEN SE-FÄLLEN}

Im Zuge der Reformdiskussion um die deutsche Unternehmensmitbestimmung wurde die Frage nach der Rolle der SE gestellt. Bisweilen wurde argumentiert, dass deutsche Unternehmen wegen der Mitbestimmung bei der Gründung von SEs benachteiligt bzw. ausgeschlossen seien. Die Verteilung der SE-Fälle widerlegt dieses Argument. Fast die Hälfte aller normalen SEs hat ihren Unternehmenssitz in Deutschland.

Möglicherweise könnte die SE aber aus anderer Richtung eine Herausforderung für das deutsche System darstellen. Sicherlich ist es zu früh für Schlussfolgerungen auf empirisch gesicherter Basis. Dennoch kann man spekulieren, warum von der Rechtsform der SE in Deutschland im Vergleich zu den anderen EU-Staaten mehr Gebrauch gemacht wird. Wird die SE möglicherweise genutzt, Arbeitnehmerbeteiligung einzufrieren oder gar einzuschränken?

Die SE scheint besonders für solche Unternehmen eine hohe Attraktivität zu haben, die für Fragen der Arbeitnehmerbeteiligung relevante Schwellenwerte erreichen. Einige Unternehmen, die an der Schwelle von 2.000 Beschäftigten stehen und damit in den Geltungsbereich des 1976er-Mitbestimmungsgesetzes gelangen, versuchen rechtzeitig, mittels einer Vereinbarung zur Arbeitnehmerbeteiligung in einer zukünftigen SE die bisherige Situation einzufrieren. Dies gilt gerade für solche Unternehmen, deren wirtschaftliche Entscheidungen ein stark ins Tagesgeschäft involvierter Inhaber trifft. Entweder wechselten sie, wie erwähnt, direkt ins monistische System oder schrieben eine geringere Arbeitnehmerbeteiligung im AR dauerhaft fest, als dies nach dem Gesetz für eine deutsche AG möglich wäre. ${ }^{10}$

In den größeren deutschen Fällen wird versucht, bei der SE-Gründung den AR zu verkleinern bzw. trotz steigender Arbeitnehmerzahl einen im Vergleich zum Recht für eine $A G$ nicht vergrößerten $A R$ beizubehalten. Ziel dieser Pläne scheint auch zu sein, die Zahl der externen Mitglieder auf Arbeitnehmer- wie auf Anteilseignerseite möglichst gering zu halten. Die Verkleinerung des AR betrifft zwar beide Seiten gleichermaßen, jedoch verlieren auf Arbeitnehmerseite vor allem hauptamtliche Gewerkschaftsvertreter im Vergleich zu Arbeitnehmervertretern aus dem jeweiligen Unternehmen an Gewicht.

Das Vorher-Nachher-Prinzip (Art. 4 SE-RL) bedeutet, dass mindestens das Ausmaß an Arbeitnehmerbeteiligung beibehalten werden muss, welches im Vorgängerunternehmen vorhanden war. Zumindest hinsichtlich der proportionalen Beteiligung scheint hier ein Mechanismus eingebaut zu sein, der Befürchtungen entgegensteht, dass die SE dazu benutzt wird, Arbeitnehmerbeteiligung einzufrieren oder gar einzuschränken.

Dass es dennoch Ansätze in diese Richtung gibt, zeigt, wie sehr es vor dem Hintergrund eines neuen institutionellen Arrangements, das Verhandlungen erfordert, auf eine Machtbalance zwischen Arbeitgeber- und Arbeitnehmerseite ankommt. Gegenmachtspositionen werden hauptsächlich seitens der Gewerkschaften aufgebaut und eingenommen, während die betrieblichen Arbeitnehmer allein nicht über die notwendigen Ressourcen verfügen; ihre Abhängigkeit von der Unternehmensleitung scheint zu groß zu sein. Die Akti-

\footnotetext{
10 So haben Conrad Electronic SE und Mensch und Maschine SE keinen Arbeitnehmervertreter in ihrem monistisch-strukturiertem board. Im Falle von Surteco wurde, trotz einer Arbeitnehmerzahl von über 2.000, eine Beibehaltung der bisherigen Drittelbeteiligung im AR vereinbart.
} 
vitäten insbesondere der Europäischen Gewerkschaftsföderationen werden weiterhin erforderlich sein, auch wenn das gerade in den kleineren Unternehmen schwer ist, in denen der gewerkschaftliche Organisationsgrad sehr niedrig ist. Umgekehrt ist es auch denkbar, dass die (deutsche) Mitbestimmung mittels des neuen institutionellen Arrangements in Länder ausstrahlen kann, in denen Arbeitnehmerbeteiligung schwächer ausgeprägt ist (Sorge 2006).

\section{Ausblick}

Dieser Beitrag kann sicherlich noch keine endgültigen Schlüsse ziehen in Bezug auf die Relevanz der SE und ihrer Auswirkungen auf die Systeme von Industrial Relations in den Mitgliedstaaten und in der EU. Zum einen ist die empirische Basis noch zu schmal; zum anderen können noch keine Aussagen gemacht werden zur Praxis der Arbeitnehmerbeteiligung in den Unternehmen.

Das institutionelle Arrangement der Arbeitnehmerbeteiligung in der SE gibt wie schon bei den EBRs - lediglich Verfahrens- und keine inhaltlich-materiellen Regelungen vor. Die nationalen Umsetzungen der SE-RL zeigen, dass wir nicht von einem einheitlichen System der Arbeitnehmerbe- teiligung sprechen können, vielmehr unterscheiden sich die 30 Umsetzungsgesetze von Mitgliedstaat zu Mitgliedstaat (Nagel 2006). Auch die Beteiligungsvereinbarungen in den ersten SEs variieren. Wahrscheinlich werden sich die Formen der Arbeitnehmerbeteiligung in Europa mit der SE weiter heterogenisieren. Dies gilt zunehmend nicht mehr nur für die Verhältnisse zwischen, sondern auch innerhalb der einzelnen Mitgliedstaaten, und mehr noch zwischen einzelnen Unternehmen/SEs. Dass mit der SE eine Harmonisierung der Arbeitnehmerbeteiligung „nach oben“ gelingen kann, erscheint ziemlich unwahrscheinlich. Vielmehr ist das institutionelle Arrangement gerade (nur) darauf angelegt, die Beteiligungsrechte, die in den Mitgliedstaaten bestehen, auch für die erste ,europäische" Rechtsform zu sichern.

Die Wirkungen gehen in zwei Richtungen: Zum einen zeigen einige, gerade deutsche Fälle, dass bestimmte Arbeitgeber mit der SE auszutesten versuchen, ob und inwieweit sich Arbeitnehmerbeteiligung einfrieren oder einschränken oder zumindest an „unternehmensspezifische Gegebenheiten" anpassen lässt; der weitestmögliche Ausschluss der Gewerkschaften scheint ein Ziel zu sein. Andererseits findet in gewisser Hinsicht eine „Ausstrahlung“ von Arbeitnehmerbeteiligung, wenn auch nur unternehmensspezifisch, dadurch statt, dass die Leitungsgremien der SEs auch auf Arbeit- nehmerseite internationalisiert werden. Wenn große und mitbestimmte Unternehmen durch die SE-Gründung einen Vorteil für sich erkennen, scheuen sie sich offenbar nicht, mit Arbeitnehmervertretern Vereinbarungen abzuschließen, die zumindest formal eine Ausweitung von Beteiligungsoptionen für Arbeitnehmer aus vielen EULändern mit gesetzlich eher schwach ausgeprägten Möglichkeiten bedeuten.

Diese Tendenzen führen zu unternehmensspezifischen Industrial Relations, die neben den tradierten nationalen Systemen (ent-)stehen. Derartige „Parallelsysteme“ könnten auch Druck ausüben auf die Gesetzgebung zu Corporate Governance und Arbeitnehmerbeteiligung in den Mitgliedstaaten. Solche Tendenzen können gerade von Unternehmen ausgehen, in denen der gewerkschaftliche Organisationsgrad niedrig ist und in denen sich insofern Verhandlungslösungen durchsetzen können, die weit von den „regulären“ nationalen Regelungen abweichen. Es bleibt weiter zu beobachten, welche Unternehmen aus welchen Gründen und mit welchen Zielen die Rechtsform der SE wählen. Zumindest für einige Fälle muss sie gewisse Vorteile bieten. Aus deutscher Sicht könnten diese auch darin bestehen, mit Formen der Arbeitnehmerbeteiligung $\mathrm{zu}$ experimentieren.

\section{LITERATUR}

Bercusson, B. (1992): Maastricht: a fundamental change in European Labour Law, in: Industrial Relations Journal 3, S. 177-190

Blanke, T. (2005): "Vorrats-SE“ ohne Arbeitnehmerbeteiligung, Düsseldorf

Blanke, T./Köstler, R. (2006): Europäische Betriebsräte-Gesetz, Europäische Mitbestimmung - SE, Baden-Baden

EMF (2003): The European Company - SE. EMF guidelines, Brüssel EWCs Bulletin (2006): European Company Statute update, in: European Works Council Bulletin 9-10, S. 12-14

Fulton, L. (2006): The forgotten resource: corporate governance and employee board-level representation, the situation in France, the Netherlands, Sweden and the UK. Report for Hans-Böckler-Foundation, London Gold, M./Schwimbersky, S. (2007): The European Company Statute: Implications for Industrial Relations in the European Union, in: European Journal of Industrial Relations (im Erscheinen)
Keller, B. (2002): The European Company Statute: Employee involvement - and beyond, in: Industrial Relations Journal 5, S. 423-444 Kerckhofs, P. (2006): European Works Councils. Facts and Figures 2006, Brüssel

Kluge, N./Stollt, M. (Hrsg.) (2006): The European company - Prospects for worker board-level participation in the enlarged EU, Brüssel Kluge, N./Stollt, M. (2007): Übersicht: Unternehmensmitbestimmung in den 27 EU-Mitgliedstaaten, in: Köstler, R. (Hrsg.): Die Europäische Aktiengesellschaft: Eine Einführung in die Europäische Aktiengesellschaft mit Anmerkungen zur grenzüberschreitenden Verschmelzung, Düsseldorf, S. 47-50

Köstler, R. (2006): Was ist eine Europäische Aktiengesellschaft?, in: Kluge, N./Stollt, M. (Hrsg.): Die Europäische Aktiengesellschaft (SE) Perspektiven für eine europäische Unternehmensmitbestimmung, Brüssel, S. 15-29 
Köstler, R. (2007): Die Europäische Aktiengesellschaft. Eine Einführung in die Europäische Aktiengesellschaft mit Anmerkungen zur grenzüberschreitenden Verschmelzung, Düsseldorf Lecher, W./Platzer, H.-W./Rüb, S. (2002): European Works Councils: Negotiated Europeanisation. Between statutory framework and social dynamics, Aldershot

Nagel, B. (2006): Der Konflikt zwischen der deutschen und angelsächsischen Corporate Governance und die Europäische Gesellschaft (SE), in: Brandes, W./Eger, T./Kraft, M. (Hrsg.): Wirtschaftswissenschaften zwischen Markt, Norm und Moral. Festschrift für Peter Weise, Kassel, S. 337-359

Nagel, B./Freis, G./Kleinsorge, G. (2005): Die Beteiligung der Arbeitnehmer in der Europäischen Gesellschaft - SE, München

Röthig, O. (2006): Allianz SE - Cornerstone of a new reference model?, in: Mitbestimmung 8, S. 54-58

Sachverständigengruppe European Systems of Worker Involvement (1997): Abschlussbericht, Brüssel

Schwimbersky, S. (2006): Worker participation in the Societas Europaea The beginning of the end of compulsory board-level representation in Europe?, Paper presented at the IREC 2006 conference "The Future of Social Models", Ljubljana, August 31st-September $2^{\text {nd }}$

Schwimbersky, S./Rehfeldt, U. (2006): Case Study Report on Plansee SE, erhältlich unter http://www.worker-participation.eu/european_company/ se_companies/case_studies
SEEurope-Network (2007): SEs in Europe - Established, in preparation, announced interest, sold Vorrats-SEs, transformed, liquidated and failed. Prepared by Sandra Schwimbersky and Melinda Kelemen, last update 08-06-2007, http://www.worker-participation.eu/european_company Sorge, A. (2006): Mitbestimmung für die Europäische Aktiengesellschaft: Nützliche Lehren aus mehr als dreißig Jahren Seifenoper, in: Beckert, J./ Ebbinghaus, B./Hassel, A./Manow, P. (Hrsg.): Transformationen des Kapitalismus, Festschrift für Wolfgang Streeck, Frankfurt/New York, S. 249-271

Theisen, M./Wenz, M. (2005): Die Europäische Aktiengesellschaft. Recht, Steuern, Betriebswirtschaft der Societas Europaea (SE), Stuttgart van het Kaar, R. (2005): Company Law and workers' interest, in: Transfer 2, S. 179-188

van het Kaar, R. (2006) Mitbestimmung in Europa: Die Bedeutung des Gesellschaftsrechts für die Vertretung von Arbeitnehmerinteressen, in WSI-Mitteilungen 11, S. 624-629

Villiers, C (2006): The Directive on employee involvement in the European Company: Its role in European corporate governance and industrial relations, in: International Journal of Comparative Labour Law and Industrial Relations 2, S. 183-211

Walton, R./McKersie, R. (1991): A behavioral theory of labor negotiations. An analysis of a social interaction system, New York 